\title{
A Multi-Layer Extension of a Bayesian Neural Network
}

\author{
Anders Holst \\ Anders Lansner \\ ahoesans.kth.se \\ alalsans.kth.se \\ SANS, NADA, Royal Institute of Technology, S-100 44 Stockholm, Sweden
}

A Bayesian neural network [2] can be seen as implementing a Bayesian classifier, which is optimal for the case of independent evidence [3]. However, the performance of a standard one-layer Bayesian network is degraded when the underlying independence assumptions are violated. One way to solve this is to turn to a multi-layer architecture, where dependencies among the inputs can be removed or, in practice, at least reduced in the internal representation.

The normal way to treat strong dependencies among the evidence is to add an intermediate layer between the input (evidence) and the output (classes) [1]. This intermediate level consists of complex units, i.e. units that combine information from the input layer. If for example the units $a$ and $b$ are correlated, we produce a complex unit $a b$ that is active when both $a$ and $b$ are active. The classes will now depend on $a, b$ and $a b$, but these three units are still not independent, and the Bayesian model can not be used directly.

Instead we propose the following procedure. We start from a coding where each event is represented with one positive and negative unit. If we now find that two of these events are dependent, we merge the two events into one, with all possible combinations of the outcomes of the old events. For example, if the two primary events $A=\{a, \bar{a}\}$ and $B=\{b, \bar{b}\}$ are not independent, we insert in their place the composite event $A B=\{a b, a \bar{b}, \bar{a} b, \bar{a} \bar{b}\}$. We call this kind of group, with all possible combinations of some primary evidence, a complex column. If we manage to divide the primary events into independent groups, we can create a complex column from each such group. The Bayesian model is then directly applicable on these complex columns.

There are reasons to try to keep the order of the complex columns low. One is that the number of complex units increases rapidly with the order. Another reason is that when the columns grow larger, the generalization capability tends to decrease. This is the well known trade-off between probabilistic accuracy and generalization. Although the estimation of probabilities by the network gets better with larger columns, more training data are required to estimate the more detailed distribution.

Above it is assumed that each primary event occurs in only one complex column. If some primary event is a member of more than one column, then these will not be independent, and the estimation of probabilities will be distorted. However, if all primary events occur in the same number of complex columns, say $m$, it is possible to compensate for this by dividing all weights by $m$. We call such a coding $m$-covering.

There are cases in which a multiple covering will allow a lower order of the columns. Consider as a simple example the case of a $2 \times 2$ lattice with all patterns consisting of two active units, divided into three classes: vertical ( 践 or 㗁), horizontal ( These classes are not linearly separable. If only a one-covering is used, a complex column of order four is required. However, if we allow a two-covering, we can solve it with only second order columns. In such situations this mechanism will thus save complexity, and at the same time increase generalization in the network.

[1] A. Lansner and Ö. Ekeberg. An associative network solving the "4-Bit ADDER problem". Proc. of the IEEE First Annual Int. Conf. on Neural Nctworks, 2:549-556, 1987. San Diego, USA.

[2] A. Lansner and Ö. Ekeberg. A one-layer feedback, artificial neural network with a Bayesian learning rule. Int. J. Neural Systems, 1(1):77-87, 1989.

[3] M. L. Minsky and S. A. Papert. Pcrccptrons. MIT Press, 1988. 